\title{
Stage IIA Pancreatic Cancer AJCC v8
}

National Cancer Institute

\section{Source}

National Cancer Institute. Stage IIA Pancreatic Cancer A/CC v8. NCI Thesaurus. Code C134925.

Stage IIA includes: T3, N0, M0. T3: Tumor measuring more than $4 \mathrm{~cm}$ in greatest dimension. N0: No regional lymph node metastasis. M0: No distant metastasis. (AJCC 8th ed.) 